\title{
High Fidelity Reconstruction of Experimental Field Ion Microscopy Data by Atomic Relaxation Simulations
}

\author{
Shyam Katnagallu ${ }^{1}$, Ali Nematollahi ${ }^{1}$, Michal Dagan ${ }^{2}$, Michael Moody ${ }^{2}$, Blazej Grabowski ${ }^{1}$, Baptiste \\ Gault $^{1}$, Dierk Raabe ${ }^{1}$ and Jörg Neugebauer ${ }^{1}$. \\ 1. Max-Planck-Institut für Eisenforschung GmbH, Max-Planck-Straße 1, Düsseldorf, Germany. \\ 2. Department of Materials, University of Oxford, Parks Road, Oxford, UK.
}

A key question in materials science is to identify how atomic scale structural and chemical motifs determine the properties of materials. To address this question, atomic-scale investigations of materials are instrumental. The huge advances made in the field of ion emission microscopy such as atom probe tomography (APT), allow today routine studies at the atomic scale. The predecessor to APT, field ion microscopy (FIM) [1] invented by E.W. Müller in 1951, was the first technique to image individual atoms on the surface of a sharp metallic needle. A tomographic FIM or 3D FIM employs a controlled field evaporation of atoms from the surface of a cryogenically cooled sharp needle-shaped specimen held at high enough electric field, while constantly imaging the field evaporating surface by an ionizing imaging gas. From a modelling perspective atomistic simulations such as density functional theory (DFT), molecular dynamics (MD) and Monte Carlo methods, nowadays also provide detailed insight into the atomic structure of materials. The current study aims to combine such atomic scale experimental investigations with corresponding atomic scale simulations with the aim to obtain specific structural atomic motif features for which the experimental reconstruction of the atomic positions alone is not precise enough. These experiment informed simulations, or simulation enhanced experiments, cannot only save computation time as the simulations are conducted directly on experimentally obtained atomic configurations but it can also qualify the FIM technique as the most precise available tool for the full tomographic and crystallographic real space imaging of atoms. The suitability of the extracted experimental atomic scale data for such informed atomistic simulations is also explored here.

An automated routine to extract the atomic coordinates from 3D FIM images taken on pure tungsten was recently proposed by M. Dagan [2]. Based on this pioneering work, a new and enhanced automated routine is proposed here to extract the coordinates from 3D FIM data without any apparent loss of data. A region of interest (ROI) is chosen in the 3D FIM images of tungsten, such that the image exhibits true atomic resolution. In this case a ROI was chosen around a $\{111\}$ pole. The first step of the automated routine is denoising and enhancing the images using a Laplacian of Gaussian filter (LoG). Then a variant of zero crossing edge detection algorithm is used to identify the positions of the atoms. An associated classification based on an entropy filter allows to assign individual atoms to their corresponding terraces in the image. The intensity at the center of the images, tracked through the entire sequence, is used to identify the number of planes evaporated. This enables building the correct depth coordinate for the identified atoms. Since an atom is imaged multiple times before its evaporation, an agglomerative hierarchical clustering algorithm is employed to track the individual atoms through these images. Finally, a 3D point cloud is built based on the coordinates of atom just before its evaporation. These steps are shown in Figure 1.

The point cloud shows deviations from the expected $\mathrm{W}$ lattice positions. This is expected because the imaged position is not the position of an atom itself, but a region in space with significant field ionization located above the surface atom. Thus, the obtained 3D point cloud data can be assumed to be 
in an excited state and a molecular relaxation procedure should allow obtaining the 'corrected' data. A calibrated and rescaled data set was enclosed in a W lattice and used as input to a molecular statics energy minimization simulation. For this purpose, the Large-scale Atomic/Molecular Massively Parallel Simulator (LAMMPS) code is deployed, making use of an embedded atom method (EAM) potential [3]. Figure 2 shows the results from the relaxation procedure. The relaxed structure correctly reproduces the small amount of vacancies. Also, deviations from the ideal crystal structure are observed in the vicinity of vacancies. These preliminary results show the potential of using a molecular relaxation procedure to get 'corrected' experimental data. An advantage of this physics based rectification method over the geometrical rectification [4] is its potential to preserve the defects in the rectified data.

In conclusion, the proposed new automated routine has the ability to identify all the atoms in the FIM image and thus no data loss is expected. This enables novel pathways to obtain valuable insights when tracking the subtle changes in the imaged positions as the atom's neighborhood evolves. Due to these features such a routine is believed to be better suited for revealing the atomic structures around extended defects such as dislocation cores and grain boundaries. The use of well-established molecular relaxation simulations as a way of obtaining physics-corrected positioning data has been explored in this analysis. Thus more precise characterization of FIM data with this technique might be a pathway to discover the specific atomistic motifs surrounding defects.

\section{References:}

[1] Müller Erwin W, Zeitschrift für Physik 131.1 (1951), p. 136.

[2] Dagan M, PhD thesis, University of Oxford (2016).

[3] Bonny G, Grigorev P, and Terentyev D, Journal of Physics: Condensed Matter 26.48 (2014).

[4] Moody Michael P, et al. Microscopy and Microanalysis 17.02 (2011), p. 226.
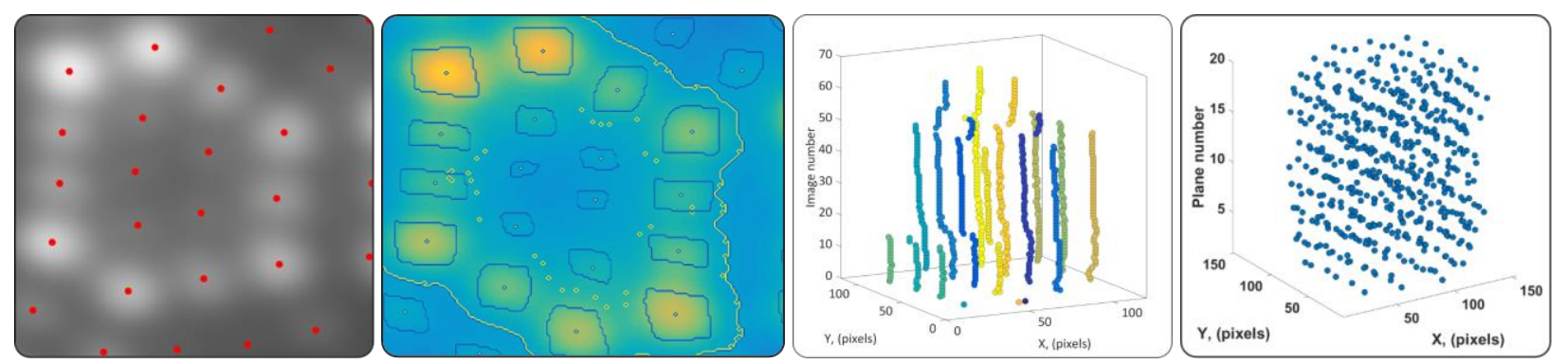

Figure 1. Various steps of the automated routine described in the text to extract data from 3D FIM images.

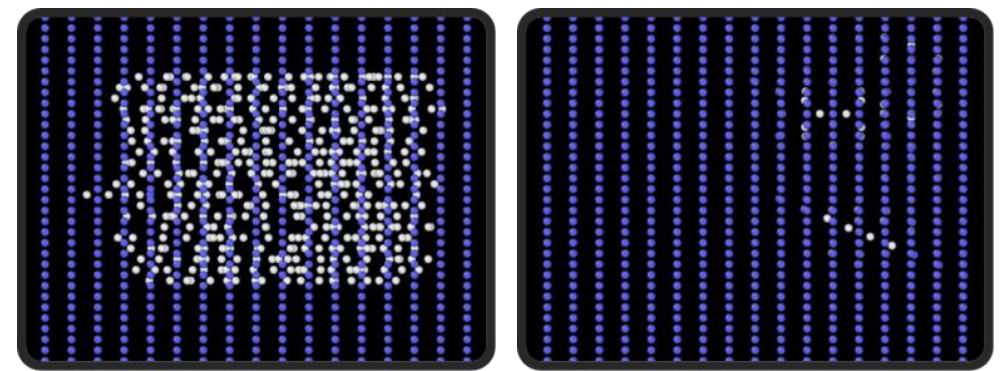

Figure 2. Before (left) and after (right) atomic configuration of the proposed molecular relaxation procedure (white spots are non-ideal positions, blue spots are the ideal positions). 